Research Article

\section{The influence of HBV or HCV infections on the pregnancy course}

\author{
Tadeusz Wojciech Lapiński*
}

Department of Infectious Diseases and Hepatology, Medical University in Bialystok, Poland

\section{Abstract}

The incidence of $\mathrm{HBV}$ infections among the pregnant in Europe falls within the range of $1 \%-7 \%$, whereas it is $1.7 \%-4.3 \%$ for $\mathrm{HCV}$.

The aim was to assess the course of pregnancy among women infected with HBV or HCV, and the condition of neonates in the fifth minute after the birth.

The study included 157 pregnant individuals infected with HBV, 53 infected with HCV, and 330 healthy pregnant women. None of the women infected with HBV and HCV as well as from the control group were infected with HIV, and none of them took intoxicants.

Weight of neonates delivered by healthy women was higher as compared with children born by women infected with HBV or HCV (3,517 vs. 3,347 and 3,366). The Apgar score of neonates delivered by women with HBV and HCV infections was lower as compared with the children born by healthy women (9.4 vs. 9.3 vs. $9.7 ; p<0.05)$. Premature births occurred more often in HBV and HCV-infected women than in the control group $(14.6 \%$ and $24.5 \%$ vs. $6.96 \%$; $p<0.05)$. Miscarriages were significantly more common among the pregnant with $\mathrm{HCV}$ infections as compared with the pregnant who were healthy $(9.4 \%$ vs. $1.8 \% ; p<0.05)$. In comparison with the healthy individuals, this group of patients experienced pruritus $(10.5 \%$ vs. $4.2 \% ; p<0.05)$, oedemas $(9.4 \%$ vs. $2.4 \% ; p<0.05)$, and hypertension $(9.4 \%$ vs. $1.5 \% ; p<0.05)$ more often.

An increase in HBV loads was observed between the 6 th and $28^{\text {th }}-32^{\text {nd }}$ week of pregnancy among the infected with HBV, and then, a decrease was observed in the 6th months after the delivery.

The pregnant infected with HBV without HBsAg (-) and the infected with HCV are subject to common incidence of premature births. Women infected with HCV often experience oedemas, hypertension, and pruritus.
More Information

*Address for Correspondence: Tadeusz Wojciech Lapiński, Department of Infectious Diseases and Hepatology, Medical University in Bialystok, Poland,

Tel; +48 604651709; Email: twlapinski@wp.pl

Submitted: 20 January 2020

Approved: 12 August 2020

Published: 14 August 2020

How to cite this article: Lapiński TW.

The influence of HBV or HCV infections on the pregnancy course. Clin J Obstet Gynecol. 2020; 3: 098-102.

DOI: 10.29328/journal.cjog.1001058

Copyright: (c) 2020 Lapiński TW. This is an open access article distributed under the Creative Commons Attribution License, which permits unrestricted use, distribution, and reproduction in any medium, provided the original work is properly cited.

Keywords: HBV or HCV infection in pregnancy; Childbirth

Abbreviations: HBV: Hepatitis B Virus; HCV: Hepatitis C Virus; HIV: Human immunodeficiency viruses; HBIG: Hepatitis B immunoglobulin; HBsAg: surface antigen of the hepatitis $B$ virus; HBeAg: Hepatitis B envelope antigen; NAs: nucleoside/Nucleotide Analogues: TDF: Tenofovir Disoproxil Fumarate

(W) Check for updates

O OPEN Access

\section{Introduction}

The incidence of HBV (Hepatitis B Virus) infections with the pregnant in Europe ranges from 1\% to 7\% [1]. Pregnant women are usually aware of the HBV infections; less often they learn about the infection upon examination performed during initial stages of the pregnancy. Neonates delivered by women infected with HBV become infected in less than $1 \%$ of cases in Europe [2]. The likelihood of a neonate becoming infected in a perinatal or postnatal way does not exceed $10 \%$ thanks to widely applied active and passive prophylaxis of children delivered by HBV-infected women (a vaccine and a HBIG (Hepatitis B immunoglobulin) specific serum administered within 12 hours after the delivery] [3,4]. It is possible for a child to become infected via an intrauterine way. Also, a child may become infected by an HBV-infected woman who has high HBV viraemia ( $>200,000 \mathrm{IU} / \mathrm{ml}$ ) or high HBsAg (surface antigen of the hepatitis B virus) concentration (> 4-4.5 $\log 10 \mathrm{IU} / \mathrm{ml}$ ) [3]. The risk of such an infection regards most often the pregnant with HBV viraemia above $6 \log 10 \mathrm{IU} / \mathrm{mL}$ and HBeAg (+) (Hepatitis B envelope antygen) presence. The likelihood of an infection with the virus increases proportionally to the viraemia increase $[4,5]$. The devised guidelines present the ways of dealing with women infected with HBV that want to become pregnant.

Table 1 Guidelines regarding the ways of dealing with pregnant women infected with HBV $[3,6]$.

Antiviral drugs used in the pregnant infected with HBV decrease the risk of infecting the foetus, however, none of the currently used NAs (nucleoside/nucleotide analogues) are listed as category A according to FDA (Food and Drug 
Table 1: Guidelines regarding the ways of dealing with pregnant women infected with HBV $[3,6]$.

$1^{\text {st }}$ trimester of the pregnancy - a test for HBsAg presence is recommended; in case of HBsAg (+) - viraemia assessment.

There is no indication to start the therapy for women without advanced fibrosis.

Women with advanced fibrosis or cirrhosis - TDF therapy is recommended.

The pregnant with high DNA HBV concentration ( $>200,000 \mathrm{IU} / \mathrm{mL}$ ) or HBsAg [ $>4 \log _{10} \mathrm{IU} / \mathrm{ml}$ ) should receive TDF in $24^{\text {th }}-28^{\text {th }}$ week of the pregnancy. It is recommended to discontinue the drug 12 weeks after the delivery.

Pregnant women that are treated with NA should continue the TDF therapy; in case of ETV or another NA treatment, the drug should be changed to TDF.

Breastfeeding is not contraindicated for women infected with HBV that are not and are treated with TDF.

Administration), and thus, their use in pregnant women should be careful. European (EASL-European Association for the Study of the Liver) and American (AASLD- American Association for the Study of Liver Diseases) guidelines allow for administering TDF between the $24^{\text {th }}$ and $32^{\text {nd }}$ week of pregnancy in special cases. The drug is used in order to decrease the risk of infecting the foetus.

The probability of a newborn being infected by an HCVinfected mother ranges from $1.7 \%$ to $4.3 \%$. It is the highest among the women that are infected with HCV/HIV (19.4\%), and also, among individuals that take intravenous intoxicants (and who are not HIV-infected; 8.6\%) [7]. The risk of infecting a neonate and a negative influence on the clinical pregnancy course of patients infected with HCV were the basis for EASL and AASLD guidelines for antiviral treatment for all women at procreative age "in the first place" (especially the ones who wanted to become pregnant) [8]. Limited studies on DAA efficacy and adverse reactions among the HCV-infected pregnant women indicate a decreased likelihood of infecting a neonate among the pregnant that take such antiviral drugs. Although these drugs have not been approved to be used in the pregnant yet, about $60 \%$ of women infected with HCV and pregnant declare that they are eager to use them in order to avoid infecting the child [9].

Despite specified procedures that concern dealing with the pregnant infected with $\mathrm{HBV}$ and $\mathrm{HCV}$, observations regarding the influence of these viruses on the course of pregnancy are ambiguous.

\section{Aim of the work}

The aim of the work was to perform a clinical assessment of the pregnancy course among women infected with HBV or HCV. The following was determined: type of delivery, neonates' condition in the fifth minute after the delivery, weight at birth, and sex. All patients had their biological parameters of liver function monitored. The pregnant infected with HBV had viraemia monitored during the course of pregnancy and after the delivery. HCV-infected women had the virus genotype and initial viraemia assessed.

\section{Material and methods}

The study included 157 pregnant women infected with HBV aged from 21 to 42 (mean age: $29 \mathrm{y} / \mathrm{o}$ ), and 53 pregnant women infected with HCV aged from 19 to 46 (mean age: 28 $\mathrm{y} / \mathrm{o}$ ). Three hundred and thirty healthy pregnant women aged from 18 to 40 (mean age: $28 \mathrm{y} / \mathrm{o}$ ), who lived in the same region as the infected women, constituted a control group.

None of the women infected with HBV and HCV as well as from the control group was HIV-infected and none of them took intoxicants.

Qualification criteria for HBV-infected women included no $\mathrm{HBeAg}$ and exclusion of cirrhosis. None of the pregnant infected with HBV had been qualified to the treatment earlier nor had been treated with antiviral drugs. The following was monitored among the HBV-infected women: viraemia, ALT activity, serum ALP within the first six weeks of pregnancy, between the $28^{\text {th }}$ and $32^{\text {nd }}$ week of pregnancy and six months after the delivery. In the group of the pregnant infected with $\mathrm{HCV}$, viraemia and virus genotype were determined in the $5^{\text {th }}$ week of pregnancy, whereas ALP and ALT activity was determined in the first 6 weeks of pregnancy, between $28^{\text {th }}$ and $32^{\text {nd }}$ week of pregnancy, and six months after the delivery.

Neonates had their clinical condition assessed in the fifth month of life by means of Apgar score.

All patients had serum HBsAg, $\mathrm{HBeAg}$, anti-HBe, and antiHCV antibodies assessed once by means of microparticle enzyme immunoassay (MEIA), and using Abbott tests (Germany).

HBV-DNA quantity was determined by RT-PCR using sets of COBAS AmpliPre/COBAS TagMan HBV Test, version 2.0 produced by ROCHE. The sensitivity amounted to $9 \mathrm{IU} / \mathrm{mL}$, whereas linearity was $20 \mathrm{IU} / \mathrm{mL}$.

Among women with positive anti-HCV, quantitative assessment and genotype were determined by RT-PCR method and using sets of COBAS HCV Test, version 2.0 produced by ROCHE (Germany).

The pregnant provided their informed consent to participate in the study according to the protocol accepted by the Bioethical Committee of Medical University in Bialystok (R-I-002/134/2019).

Statistical analysis of data was conducted by using STATISTICA.PL produced by StatSoft for Windows 10 operating system. The study used Mann-Whitney U test, Spearman's rho, student's t-test, and chi-squared test $(\chi 2)$. The level of significance was set at $p<0.05$. 


\section{Results}

Mean pregnancy duration in HBV- and HCV-infected as well as among the healthy patients was similar and amounted to 38-39 weeks. The frequency of spontaneous labour was comparable and amounted to $52 \%$ for HBV patients, $42 \%$ for $\mathrm{HCV}$ patients, and $58 \%$ in the control group.

Weight of children delivered by healthy women was higher as compared with children delivered by HBV- or HCV-infected females $(3,517$ vs. 3,347 vs. 3,366). In case of HBV-infected women, the weight was significantly lower as compared with the healthy women (Figure 1).

Having assessed the Apgar score at the fifth minute of life, neonates of HBV- and HCV-infected women showed lower scores as compared with children born to healthy females (9.4 vs. 9.3 vs. 9.7; $p<0.05$ ), (Figure 2).

Premature births occurred significantly more often in the HBV- and HCV-infected as compared with the control group (6.96\% vs. $14.6 \%$ and $24.5 \%$; $p<0.05$ ). In the group of HCVpatients miscarriages were significantly more common as compared with the healthy women $(9.4 \% v s .1 .8 \%$; $p<0.05)$. Among the HBV-patients miscarriages were more frequent than in the control group, however, it was not statistically significant.

Table 2 Characteristics of pregnant women, pregnancy and basic data on newborns.

Nausea was the most commonly observed adverse reaction in all of the pregnant women. HCV-patients significantly more often experienced pruritus ( $10.5 \%$ vs. $4.2 \%$; $p<0.05)$, oedemas (9.4\% vs. 2.4\%; $p<0.05)$, and arterial hypertension $(9.4 \%$ vs. $1.5 \% ; p<0.05)$ as compared with the healthy women.

Table 3 Symptoms that occurred in pregnant women during pregnancy.

Among the HBV-patients, an increase in viraemia between the $6^{\text {th }}$ and $28^{\text {th }}-32^{\text {nd }}$ week of pregnancy was confirmed in $46 \%$ of patients, a decrease in $15 \%$ of patients, and in $39 \%$ of cases there were no changes. In $90 \%$ of women without detectable viraemia, in the $6^{\text {th }}$ week of pregnancy, its increase was not confirmed between the $28^{\text {th }}$ and $32^{\text {nd }}$ week of pregnancy. (Figure 3).

Among the HBV-patients, an increase in viraemia was observed between the $6^{\text {th }}$ and $28^{\text {th }}-32^{\text {nd }}$ week of pregnancy. The highest increase was confirmed among the pregnant with initial viraemia of HBV DNA $\leq 4 \log 10 \mathrm{IU} / \mathrm{mL}$ in the $6^{\text {th }}$ week of pregnancy.

In 4 HBV-patients, who experienced a miscarriage, $\mathrm{HBV}$ viraemia was at $4 \log 10 \mathrm{IU} / \mathrm{mL}$, whereas in 2 it was undetectable.

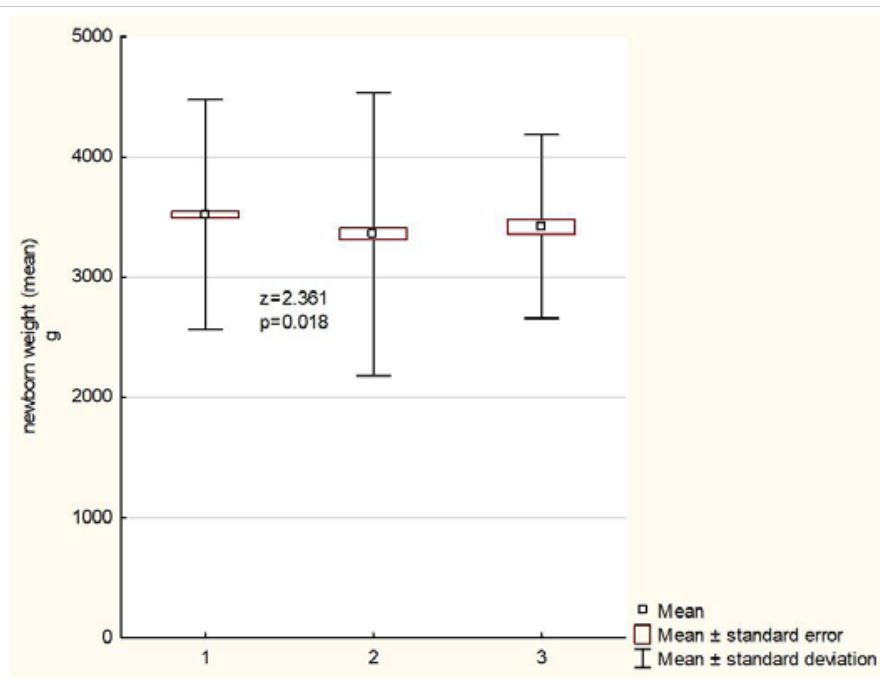

Figure 1: Neonatal weight (mean) among the examined patients and the control group. - pregnant women healthy; 2 - pregnant women infected with HBV

3 - pregnant women infected with HCV

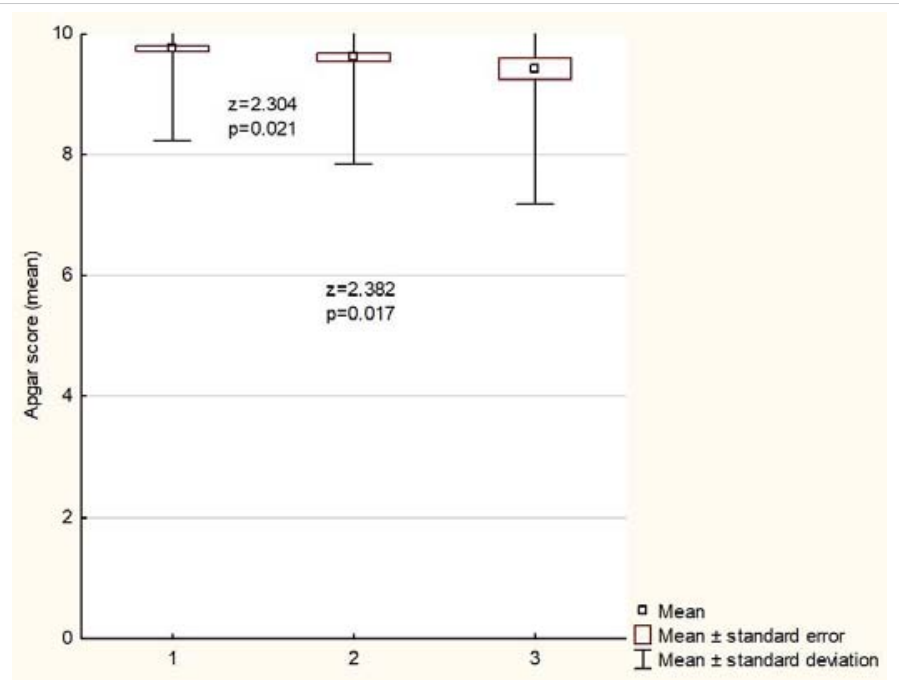

Figure 2: Neonatal scores (mean) on the APGAR scale (5 minutes) obtained in the examined groups of newborns.

- pregnant women healthy; 2 - pregnant women infected with HBV

- pregnant women infected with HCV

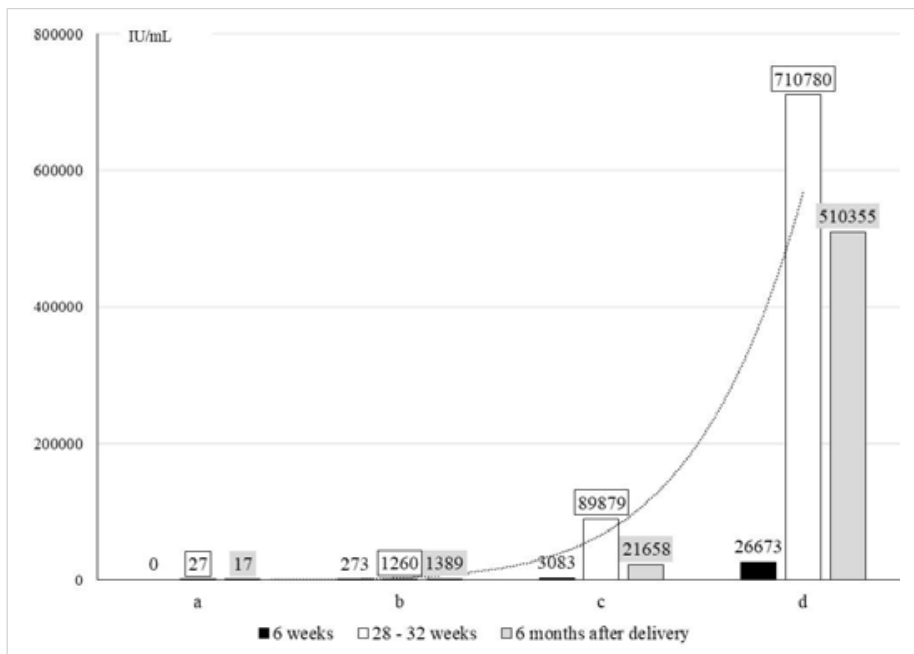

Figure 3: HBV load in serum at 6, 28-32 weeks of pregnancy and 6 months after delivery. a - pregnant with HBV DNA = 0; b - pregnant with HBV DNA $\leq 2$ Log10 IU / mL c - pregnant with HBV DNA $\leq 3$ Log10 IU / mL; d - pregnant with HBV DNA $\leq 4 \log 10 \mathrm{IU} / \mathrm{mL}$ 


\begin{tabular}{|c|c|c|c|}
\hline & \multirow{2}{*}{ pregnant healthy } & \multicolumn{2}{|c|}{ pregnant infected } \\
\hline & & HBV & HCV \\
\hline$n$ & 330 & 157 & 53 \\
\hline Age, average in years (from ... to) & $28 ;(18-41)$ & $29 ;(21-42)$ & $29 ;(20-46)$ \\
\hline Date of delivery, week (from ... to) & $39 ;(36-42)$ & $38 ;(28-42)$ & $38 ;(30-41)$ \\
\hline Premature delivery $\mathrm{n}(\%)$ & $23(6,96 \%)^{1,2}$ & $20(14,6 \%)^{1}$ & $13(24,5 \%)^{2}$ \\
\hline Miscarriage & $6(1,8 \%)^{1}$ & $6(3,8 \%)$ & $5(9,4 \%)^{1}$ \\
\hline Perinatal death & 1 & 0 & 0 \\
\hline Childbirth through the ways of nature & $194(59 \%)$ & $82(52 \%)$ & $22(42 \%)$ \\
\hline Child's sex $+/ \widehat{O}$ & 174/156; (53\%/47\%) & $73 / 84 ;(46 \% / 54 \%)$ & $29 / 24 ;(55 \% / 45 \%)$ \\
\hline Birth weight of a child (g) & 3517 & 3347 & 3366 \\
\hline Appgar scoring in 5 minutes & 9,7 & 9,4 & 9,3 \\
\hline
\end{tabular}

Table 3: Symptoms that occurred in pregnant women during pregnancy.

\begin{tabular}{|c|c|c|c|}
\hline \multirow{2}{*}{ Symptoms } & \multirow{2}{*}{ pregnant healthy } & \multicolumn{2}{|c|}{ pregnant infected } \\
\hline & & HBV & $\mathrm{HCV}$ \\
\hline$n$ & 330 & 157 & 53 \\
\hline nausea n (\%) & $20(6,1 \%)$ & $10(6,4 \%)$ & $2(3,8 \%)$ \\
\hline edema n (\%) & $8(2,4 \%)^{*}$ & $8(5,1 \%)$ & $5(9,4 \%)^{*}$ \\
\hline hypertension n (\%) & $5(1,5 \%)^{*}$ & $6(3,8 \%)$ & $5(9,4 \%)^{*}$ \\
\hline diabetes $\mathrm{n}(\%)$ & $4(1,2 \%)$ & $3(1,9 \%)$ & $2(3,8 \%)$ \\
\hline urinary tract infection $\mathrm{n}(\%)$ & $2(0,6 \%)$ & $6(3,8 \%)$ & $1(1,9 \%)$ \\
\hline itchy skin n (\%) & $14(4,2 \%)^{*}$ & $11(7,0 \%)$ & $6(10,5 \%)^{*}$ \\
\hline anemia n (\%) & 0 & $4(2,5 \%)$ & 0 \\
\hline HELLP syndrome $n(\%)$ & 0 & 0 & 1 \\
\hline
\end{tabular}

HCV infections were cased in $67 \%$ of patients by $1 \mathrm{~b}$ genotype, in $20 \%$ - 3a, and in $13 \%$ - 4 genotype.

An influence of HCV RNA viraemia on possible clinical symptoms in the pregnant females was not observed.

In HBV- and HCV-patients, a mean ALT and ALP activity during the pregnancy did not change and remained within normal limits.

There were no cases of infecting a neonate both with regard to $\mathrm{HBV}$ and $\mathrm{HCV}$.

\section{Discussion}

Patients with chronic HBV infections in Europe and the US are usually characterized by the lack of HBeAg. The situation is different in Asia, and it may exert a significant influence on the course of this infection among the pregnant.

HBV and HCV infections have an effect on an increased activity of proinflammatory cytokines: IL-2, IL-6, IL-10, macrophage migration inhibitory factor (MIF), and TNF- $\alpha$. In pregnant women they may cause an increase in the percentage of miscarriages, premature births, and a worse clinical condition of delivered neonates that is determined by the Apgar score [10]. Cui, et al. compared 513 pregnant women that suffered from chronic HBV infections with 20,491 pregnant women without this infection, and showed a statistically more frequent occurrence of miscarriages in HBV-patients that were pregnant [5]. In the authors' studies, premature births were observed more often in HBV- and
HCV-patients. However, the frequency of miscarriages was statistically higher among HCV-patients as compared with the healthy individuals. Although miscarriages were often observed in HBV-patients, there was no statistically significant difference as compared with the healthy females.

During pregnancy, high concentration of adrenal corticosteroids may influence the increase in HBV viraemia $[11,12]$. In most pregnant women with HBV infections and absent HBeAg, viraemia is stable. However, in some patients an increase in HBV-DNA and ALT activity is observed during the late pregnancy and in the postnatal period [13]. In the authors' studies, an increase in viraemia was observed at the turn of the $2^{\text {nd }}$ and $3^{\text {rd }}$ trimester, and then, a decrease was observed during the $6^{\text {th }}$ month after the delivery. A significant increase with $2 \log 10$ was observed among the patients with HBV-DNA $\leq 4 \log 10 \mathrm{IU} / \mathrm{mL}$ in the $6^{\text {th }}$ week of pregnancy. In other pregnant females, a worrying increase in viraemia was not confirmed. Occasionally, during the perinatal period a significant HBV reactivation, and a serious liver damage with encephalopathy and hepatic coma may occur [14]. Among such patients, an occurrence of disseminated intravascular coagulation (DIC), hepatorenal syndrome, brain oedema, and bile duct infections were observed. Interestingly, prophylactic administration of $\mathrm{NA}$ from the $2^{\text {nd }} / 3^{\text {rd }}$ pregnancy trimester does not decrease the risk of liver failure, although it prevents a child from becoming infected [15]. Miscarriages were observed in 6 pregnant women infected with HBV. However, in none of them an HBV-DNA viraemia was confirmed to exceed $3 \log 10 \mathrm{IU} / \mathrm{mL}$. 
Having assessed the incidence of adverse events, a more common occurrence of oedemas, hypertension and pruritus was confirmed in HCV-patients as compared with the healthy ones. Dibba, et al. assessed the effects of HCV infections on the pregnant women and showed the role this infection played in occurrence of metabolic disorders of lipid and carbohydrate balance. It may have an influence on hepatic steatosis, appearance on insulin-resistant diabetes, and intensification of atherosclerotic processes. Seldom are clinical consequences of HCV infections with regard to the course of pregnancy described. An influence of simultaneous $\mathrm{HCV}$ and HIV infections or patients addicted to intoxicants do not constitute a good comparative material. In the authors' studies, a frequent incidence of oedemas, hypertension, and pruritus was confirmed among the HCV patients. These observations are difficult to explain. A significant difference in the clinical course of the pregnancy between the healthy and HBV-infected women was not confirmed. Cai, et al. observed a more common incidence of cholestasis of pregnancy among the HBV-patients. Yet, their studies were conducted on a group of HBeAg (+) patients, what constitutes a significant difference as compared to the authors' studies.

None of the delivered babies was confirmed to have HBV or HCV infection, as well as congenital defects.

\section{Conclusion}

Pregnant women infected with HBV without HBsAg, and HCV-infected females are subject to frequent premature deliveries. An increase in viraemia during the pregnancy with its consequent decrease after the delivery is observed in the pregnant with HBV and without HBeAg. HBV infections do not exert an influence on the clinical pregnancy course, whereas HCV infections may lead to oedemas, arterial hypertension, and pruritus during the pregnancy course.

All authors have met the criteria for authorship and have participated sufficiently in the work to take responsibility for it.

The manuscript has been submitted with the full knowledge and approval of the institutions given as the affiliation of the authors.

\section{References}

1. Jonas MM. Hepatitis B and pregnancy: an underestimated issue. Liver Int 2009: 133-139.

PubMed: https://pubmed.ncbi.nlm.nih.gov/19207977/
2. Komatsu $H$, Inui A. Hepatitis B virus infection in children. Expert Rev Anti Infect Ther. 2015; 13: 427-450.

3. European Association for the Study of the Liver. EASL 2017 Clinical Practice Guidelines on the management of hepatitis B virus infection. J Hepatol. 2017; 67: 370-398.

4. Patton $\mathrm{H}$, Tran TT. Management of hepatitis $\mathrm{B}$ during pregnancy. Nat Rev Gastroenterol Hepatol. 2014; 11: 402-409. PubMed: https://pubmed.ncbi.nlm.nih.gov/24686270/

5. Cui AM, Cheng XY, Shao JG, Li HB, Wang XL, et al. Maternal hepatitis $B$ virus carrier status and pregnancy outcomes: a prospective cohort study. BMC Pregnancy Childbirth. 2016; 26; 16: 87. PubMed: https://pubmed.ncbi.nlm.nih.gov/27113723/

6. Ayoub WS, Cohen E. Hepatitis B Management in the Pregnant Patient: An Update. J Clin Transl Hepatol. 2016; 4: 241-247.

PubMed: https://www.ncbi.nlm.nih.gov/pmc/articles/PMC5075007/

7. Floreani A. Hepatitis C and pregnancy. World J Gastroenterol. 2013; 190: 6714-6720.

PubMed: https://pubmed.ncbi.nlm.nih.gov/24187446/

8. Gowda C, Kennedy S, Glover C, Prasad MR, Wang L, et al. Enhanced identification of maternal hepatitis $C$ virus infection using existing public health surveillance systems. Paediatr Perinat Epidemiol. 2018; 32 : 401-410.

PubMed: https://pubmed.ncbi.nlm.nih.gov/29972246/

9. Kushner T, Cohen J, Tien PC, Terrault NA. Evaluating Women's Preferences for Hepatitis C Treatment during Pregnancy. Hepatol Commun. 2018; 2: 1306-1310.

PubMed: https://pubmed.ncbi.nlm.nih.gov/30411077/

10. Safir A, Levy A, Sikuler E, Sheiner E. Maternal hepatitis B virus or hepatitis $C$ virus carrier status as an independent risk factor for adverse perinatal outcome. Liver Int. 2010; 30: 765-770.

PubMed: https://pubmed.ncbi.nlm.nih.gov/20214739/

11. He Q, Song $X$, Huang $Y$, Huang $W$, Ye B, et al. Dexamethasone Stimulates Hepatitis B Virus (HBV) Replication Through Autophagy. Med Sci Monit. 2018; 24: 4617-4624.

PubMed: https://www.ncbi.nlm.nih.gov/pmc/articles/PMC6064191/

12. Shimizu I, Kohno N, Tamaki K, Shono M, Huang HW, et al. Female hepatology: favorable role of estrogen in chronic liver disease with hepatitis B virus infection. World J Gastroenterol. 2007; 13: 4295-4305. PubMed: https://www.ncbi.nlm.nih.gov/pmc/articles/PMC4250853/

13. Söderström A, Norkrans G, Lindh M. Hepatitis B virus DNA during pregnancy and post partum: aspects on vertical transmission. Scand J Infect Dis. 2003; 35: 814-819.

PubMed: https://pubmed.ncbi.nlm.nih.gov/14723355/

14. Yang YB, Li XM, Shi ZJ, Ma L. Pregnant woman with fulminant hepatic failure caused by hepatitis B virus infection: a case report. World J Gastroenterol 2004; 10: 2305-2306.

PubMed: https://www.ncbi.nlm.nih.gov/pmc/articles/PMC4724988/

15. ter Borg MJ, Leemans WF, de Man RA, Janssen HL. Exacerbation of chronic hepatitis B infection after delivery. J Vir Hepat. 2008; 15: 37-41. PubMed: https://pubmed.ncbi.nlm.nih.gov/18088243/ 